\title{
A prospective study to evaluate the efficacy of Total Knee Arthroplasty conducted at KLE's hospital and MRC Belgaum
}

\author{
Dr Sayaji Vishwasrao Bhamre ${ }^{1}$, Dr Putti BB ${ }^{2}$ \\ ${ }_{1,2}$ (KLE's hospital And MRC,Belgaum, India)
}

\begin{abstract}
A Total knee replacement is an established procedure in management of osteoarthritis and rheumatoid arthritis. we are presenting our experience with this procedure 24 patients underwent TKA at KLE's hospital Belgaum between august 1999 to august 2001, of these three patients underwent bilateral TKR(total 27). The main indication for TKR was OA(17 patients) followed by RA(6 patients and one pateint with post traumatic $O A$. the follow up period ranged from 6 months to 2 years. Pre operatively each patient assessed clinically and radiologically. The functional disability was recorded according to knee scociety clinical rating system in terms of pain,raange of motion,instability and functional capacity. genu varus was present in 24 patients, maximum correction being 40 degree,fixed flexion deformity in 7 patients maximum was 40degree. Knee instability was present in 2 patients and extension lag in 5 patients. The mean preoperative score was 45 which improved to 90 after surgery. A score of 85-100 represented excellent result. the complications included periprosthetic fracture of femur during manipulation for post-op stiffnessof joint in one patient and foot drop following surgery in one patient which recovered within 4 weeks. On radiological examination over all alignment averaged 4.9 valgus, mean femoral angle was 95 degree and mean tibial angle 90 degree. Mean femoral flexion was 12 degree. Mean lateral tibial angle 87.5 degree. no radiolucencies seen after two year follow up. This report reveals $81.5 \%$ excellent, $14 \%$ good And $3.7 \%$ fair clinical result. there were no poor results.
\end{abstract}

Keywords: Total knee arthroplasty

\section{Introduction}

Total knee replacement is an established procedure in management of osteoarthritis and rheumatoid arthritis. we are presenting our experience with this procedure .24 patients underwent TKA at KLE's hospital Belgaum India between august 1999 to august 2001, of these three patients underwent bilateral TKR(total 27).

\section{Aims And Objectives:}

1) To evaluate the efficacy of total knee replacement in terms of relief of pain, range of motion and stability of the joint

2)To study various complications of total knee arthroplasty

3)To compare our results with other standard series

\section{Methods And Material:}

27 total knee arthroplasties were performed in 24 patients at KLES Hospital and MRC Belgaum, India from august 1999 to august 2001.

The age of the patient varied from 48 to 80 years. There were 16 females and 8 male patients. Out of 24 patients 17 had severe OA, 6 were having RA and one patient had post traumatic OA knee. 3 patients underwent bilateral tKA at interval of three to four months. Pre operatively each patient assessed clinically and radiologically. The functional disability was recorded according to knee scociety clinical rating system in terms of pain,range of motion,instability and functional capacity. As per knee scociety clincal rating system, $95 \%$ patients had moderate to severe pain, with average of knee flexion of 90 degree. 2 patients had instability of knee joint in both anteroposterior(less then $5 \mathrm{~mm}$ ) and mediolateral(6-9 degree). Fixed flexion deformity was present in 7 patients, 5 patients had $10^{\circ} \mathrm{FFD}, 15^{\circ} \mathrm{FFD}$ and $40^{\circ} \mathrm{FFDeach}$ one patient. Extension lag of $10^{\circ}-20^{\circ}$ was seen in 5 patients. Total 24 knees had varus deformity out of which 9 had $30^{\circ}$ varus, 11 had $20^{\circ}$ varus, 3 had $10^{\circ}$ varus and one patient had $40^{\circ}$ varus deformity of the knee. The maximum varus deformity corrected measured $40^{\circ}$. Functionally $90 \%$ of patients were unable to walk more than 5 blocks and climb stairs.

The average preoperative knee society clinical score was 50 and functional score was 44 . Spinal anaesthesia was given in 10 patients and general anaesthesia in 17 patients. The arthroplasties were performed under tourniquet control. Intra operative thromboprophylaxis with parental injectionfragine2500 IUwas give.the standard medial parapatellar approach was used. Adequate soft tissue release and soft tissue balancing was done prior to making bone cuts. Medial collateral ligament repair done in two patients. The implant used were posterior stabilized INSALL BURSTEIN II modular knee system. Average size of implant was, femoral 
component $56 \mathrm{~mm}$ with tibial conponenet $56 \mathrm{~mm}$ with tibial insert of size $56 \times 10 \mathrm{~mm}$ and patellar size $32 \mathrm{~mm}$. standart postoperative physiotheraphy protocol was followed from day 1 . The patient were followed up at 3 weeks, 6 weeks, 12 weeks, 6 months, 1 year and then 6 monthly.

In each follow up visit patients were assessed by using knee society clinical rating system. Radiologicla assessement was done according to knee soceity roentgenographic evaluation by taking anteroposterior and lateral views of knee joint to assess alignment of joint and implant loosening.

Statistical analysis used: The knee scociety clinical rating system

\section{Results:}

In the 27 knees, the average preoperative knee society clinical score was 49 which improved to 89 and knee society functional score was 43 which improved to 75 .

Knee society score of 85 to 100 represented excellent result. 22 knees (81.5\%) were rated excellent, 4 knees $(14.81 \%$ as good, and one knee(3.7\%) as fair. There were no poor result.

Pre operatively all patients had moderate to severe pain. postoperatively $60 \%$ patients had mild pain at 3 weeks. At 6 weeks postoperatively only 10\% patients had mild discomfort.

With knee society clinical rating system a knee score of 85 or more points were close to normal, taking into account factors such as age and general state of of the patient's health. Thus knees with an excellent rating were painless, stable, with at least $90^{\circ}$ of flexion and did not in themselves limit the patient's activity. In some other older patients, a slight limitation in walking distance or the need to hold a rail when stair climbing was compatible with an excellent rating. However, more severe liitaion of function, whatever the cause, as well as any deficiencies in the arthroplasty itself resulted in a lower rating.

In addition normal function was classified as, the ability to walk unlimited distance, climb up and down stairs normally without support and transfer from a chair without support.

Four knees had a good result, that is a score of 70 to 84 points. Two patients had collapsed bone graft of medial condyle with mild pain and difficulty with climbing stairs. Two patients had postoperative stiffness of knee joint, which required manipulation under general anaesthesia and contributed for reduced functional score. This over all function was again limited by age and other symptomatic knee.

One patient $(3.7 \%)$ with a fair result had a preoperative diagnosis of osteoarthritis with gross osteoporosis and developed knee stiffness postoperatively due to poor patient compliance during the rehabilitation period. For which patient had close manipulation under GA. Due to gross osteoporosis patient had periprosthetic fracture of femur (Neer's type II) which was treated with close reduction andimmbobilization with $\mathrm{A} / \mathrm{K}$ cast for 8 to 10 weeks leading to impaired functional capacity in terms of inability to walk unlimited, inability to climb up and down stairs nermally and range of moveent of knee joint less than $90^{\circ}$.

17 patients $(71 \%)$ could walk an unlimited distance and climb up down stairs normally without supports. 6 patients $(25 \%)$ could climb stairs with support and one patient could not climb the stairs with involved knee.

The average preoperative range of motion was $90^{\circ}$ and the average postoperative range of motion was $110^{\circ} .18$ knees had flexion of $115^{\circ}$ or more, the maximum being $125^{\circ}$. Two knees had less than $90^{\circ}$. one knee had minor postoperative flexion contracture.

\section{Complications:}

There was one patient with peroneal nerve neuropraxia postoperatively with associates foot drop which was treated with foot drop slint and it recovered within 6 weeks.

One patient sustaiened periprosthetic supracondylar fracture of femur(Neer's typeII) while doing manipualtion under GA. Complications like DVT were absent due to thromboprophylaxis with S/C inj fragmin $2500 \mathrm{IU}$ for atleast one week post-op. Other complications like infection, vascular injury, skin necrosis,fat embolism, joint instability, patellar fracture wer absent.

Radiologically in anteroposterior view, the tibial bone cement and cement implant interface were assessed for the presence of radiolucent areas, line of reactive sclerotic bone or both. Femoral tibila angles were measured.

In lateral view, femoral flexion, tibial angle, femoral component bone interface and patellar interface were assessed. Till the last follow up there were no radiolucent lines present beneath both tibial and femoral component. long term complications like implant failure, loosening, polythene wear can not be commented due to short duration of the study.

Maximum varus deformity correction was $40^{\circ}$, FFD being corrected was $40^{\circ}$. The overall alignment of the knee postoperatively was $4.9^{\circ}$ valgus. mean femoral angle was $95^{\circ}$ and mean tibial angle $90^{\circ}$. Mean femoraal flexion was $12^{\circ}$ and mean lateral tibila angle was $87.5^{\circ}$.

This study reveals $81.5 \%$ excellent, $14.81 \%$ good and $3.7 \%$ fair result. There wer no poor results. 
Compare to other studies conducted byKrray et a, Rand, Rosenberg, bergman et al this study has shown comparable results.

\section{Figures And Tables}

Total pain, range of motion, stability score.

\begin{tabular}{|l|c|c|}
\hline Study & Preoperative mean & Postoperative mean \\
\hline Our study & 49 & 89 \\
\hline Krray et al & 32 & 93 \\
\hline Rand et al & 35 & 84 \\
\hline Rosenberg et al & 36 & 90 \\
\hline Bergman et al & 43 & 83 \\
\hline
\end{tabular}

Total functional score(knee society functional score) preoperatively it was 43 which improved to 75

\begin{tabular}{|l|c|c|}
\hline Study & Preoperative mean & Postoperative mean \\
\hline Our study & 43 & 75 \\
\hline Krray et al & 47 & 69 \\
\hline Rand et al & 49 & 86 \\
\hline Rosenberg et al & 52 & 92 \\
\hline
\end{tabular}

\section{Discussion:}

The posterior stabilized Insall Burstein II knee prosthesis has enjoyed a proven track record of excellent results in total knee arthroplasty, This impression is further documented by the fact that $81 \%$ of our patient scored 85 points or better for a rating of excellent by knee society scoring system.

In 1978, Insall-burstein posterior cruciate substituting design was developed to improve stair climbing ability, range of motion and to prevent posterior subluxation. Insall Burstein et al reported in 1978 with IB II design that 76 percent of the patients with posterior stabilized prosthesis could walk unlimited distance and climb stairs normally without support. in this study, $71 \%$ of the patients had ability to walk unlimited and could climb stairs normally without support.

The range of motion of the knee with a posterior stabilized IB II design was improved from average $90^{\circ}$ to $110^{\circ}$. Insall et al in 1978 reported in his study that the posterior stabilised implant was the only prosthesis that resulted in substantial improvement over the preoperative renge of motion(an average of $19^{\circ}$ ).

It was a consideration around 1979-80 that the ideal alignment of prosthesis should be $0^{\circ}$, a relative varus alignment. But it has been well proved that the ideal alignment should be between $5^{\circ}-10^{\circ}$ of valgusby Insall Burstein et al in 1978. In this study the overall average alignment of prosthesis was $4.9^{\circ}$ valgus.

It has been recognized that the patella should routinely be resurfaced in RA, the practice of replacing it in OA patient is still somewhat controversial. In this study all 6 aptients with RA underwent patellar resurfacing.

The success of TKA depends not only on surgical technique, prosthesis and material design but also on patient selection and a good rehabilitation programme. In this study the goal of rehabilitation programme were-

- Prevention of hazards of bed rest like DVT, pulmonary embolism and pressure sores etc

- To acheive adequate and functional range of movement.

- To train in functional daily living activities.

A rehabilitation specialist involved in preoperative planning and patient selection can influence the outcome of the surgery. In our study we carried out extensive preoperative selaection and planning with postoperative extensive rehabilitation programme to achieve above mentioned goals which resulted in $81.5 \%$ excellent results.

There were no rediolucencies about the tibial and femoral component at the end of two years follow up perhaps the study is too short for these complications to make their presence felt.

The overall incidence of DVT after TKA without any form of mechanical or pharmaceutical prophylaxis has been reported from $40 \%$ to $88 \%$. The risk of a asymptomatic pulmonary embolism may be as high as $10 \%$ to $20 \%$ with symptomatic PE reported in $0.5 \%$ to $3 \%$ of patints and amortality rate of $2 \%$. In this study we started prophylaxis LMWH as a result there were no incidence of DVT or PE in a single patient.

However we had one pateint with neuropraxia of peroneal nerve, which occured primarily with correction of FFD of knee. the incidence of peroneal nerve palsy in swedish knee arthroplasty project was $1.8 \%$ in Rheumatoid arthritis patients.

Supracondylar fractures occur infrequently after $\operatorname{TKA}(0.2 \%$ to $1 \%)$. Reported risk factors include anterior femoral nothching, osteoporosis, rheumatoid arthritis, poor flexion, revision arthroplasty, rheumatoid arthritis, 
revision arthroplasty and neurological disorders. We had one patient with periprosthetic fracture while doing manipulation under GA for poor flexion which was a result of patient's poor physiotherapy compliance with osteoporosis in elderly patient accounted for fair result.

Rand et al 1991, concluded that the most favourable variables for better and prolonged survival of TKA are, a primary arthroplasty, a diagnosis of rheumatoid arthritis and age of sixty years or more with a posterior stabilized knee prosthesis

\section{References:}

[1]. Aaron P.K., Scott R, Supracondylar fracture of the femur after total knee arthroplasty, Clin. Orthop.,219:136,1987

[2]. Andriacchi T.P., Galante j.o. et al, The influence of toatal knee replacement design on walkind and stair climbing, JBJS, 64A:1328,1982.

[3]. Asp JP, Rand JA: peroneal nerve palsy after total knee arthroplasty, Clin, Orthop. 261:233,1990.

[4]. Ecker F.C et al., The knee society total knee arthroplasty roentgenographic evaluation and scoring system, Clin. Orthop., 248: 9,1989 .

[5]. Insall JN, Dorr LD, et al., The rational of knee society clinical rating system. Clin Orthop., 248:13,1989.

[6]. Insall JN, Lachiewicz PE, Burstein AH et al., the posterior stabilized condylar prosthesis, A modification of the total condylar design,JBJS, 64-A:1982

[7]. Rand J. Ilstrup DM: Surviorship analysis of total knee arthroplasty, JBJS, 73-A: 397, 1991

[8]. Rosenberg A.G., Verner JJ et al., Clinical result of total knee arthroplasty using total condylar III prosthesis, Clin. Orthop.,273:83,1991. 